\title{
Wilson Fermions and Axion Electrodynamics in Optical Lattices
}

\author{
A. Bermudez, ${ }^{1}$ L. Mazza, ${ }^{2}$ M. Rizzi, ${ }^{2}$ N. Goldman, ${ }^{3}$ M. Lewenstein, ${ }^{4,5}$ and M.A. Martin-Delgado ${ }^{1}$ \\ ${ }^{1}$ Departamento de Física Teórica I, Universidad Complutense, 28040 Madrid, Spain \\ ${ }^{2}$ Max-Planck-Institut für Quantenoptik, Hans-Kopfermann-Strasse 1, 85748 Garching, Germany \\ ${ }^{3}$ Center for Nonlinear Phenomena and Complex Systems - Université Libre de Bruxelles, 231, Campus Plaine, B-1050 Brussels, Belgium \\ ${ }^{4}$ ICFO-Institut de Ciències Fotòniques, Parc Mediterrani de la Tecnologia, E-08860 Castelldefels (Barcelona), Spain \\ ${ }^{5}$ ICREA - Institució Catalana de Recerca i Estudis Avançats, 08010 Barcelona, Spain
}

\begin{abstract}
We show that ultracold Fermi gases in optical superlattices can be used as quantum simulators of different types of relativistic lattice fermions in 3+1 dimensions. By exploiting laser-assisted tunneling methods, we find the atomic analogue of the so-called naive Dirac fermions, and thus provide a physical realization of the fermion doubling problem. Moreover, we show how to implement Wilson fermions, and discuss how their mass can be inverted by tuning the laser intensities. In this regime, our atomic gas corresponds to a remarkable phase of matter where Maxwell electrodynamics is replaced by axion electrodynamics: a 3D topological insulator.
\end{abstract}

PACS numbers: 37.10.Jk, 67.85.-d,11.15.Ha,71.10.Fd

The formulation of relativistic fermions in lattice gauge theories (LGTs) [1] is hampered by the fundamental problem of species doubling [2], namely, the rise of spurious fermions that modify the physics at long wavelengths. To prevent the abundance of fermion doublers, a suitable tailoring of their masses is required, leading to the so-called Wilson fermions [3]. A different, but also fundamental, hindrance in high-energy physics is the strong CP problem, more precisely, the lack of experiments confirming the charge-parity violation in strong interactions [4]. To reconcile theory and experiment, the Peccei-Quinn mechanism postulates a new particle, the axion [5], whose detection still remains elusive. Interestingly enough, these two seemingly unrelated problems turn out to be closely connected. Indeed, Wilson fermions with an inverted mass give rise to a certain axion background $[6,7]$. In this Letter, we suggest to exploit this connection to explore axion electrodynamics [8] in a tabletop experiment of ultracold atoms. The exquisite and genuine control over quantum systems inherent to ultracold-atom technologies [9] allows us to propose the implementation Wilson fermions in optical superlattices (Fig. 1a). This experiment would trace a promising route to design, control, and probe the rich physics of axions. As a first step, inverting the Wilson mass via laser-assisted tunneling enables us to realize a background axion field $\theta=\pi$, which corresponds to a new class of unconventional states of matter: 3D topological insulators (TIs) $[6,7,10,11]$. These gapped phases have conducting edges protected by topological order, but respect time-reversal symmetry. We show that our proposal constitutes the first fully-controllable quantum simulator (QS) [12] of 3D TIs preserving a general anti-unitary symmetry. Besides, the space- and time-dependence of the axion field can be experimentally tailored to test the magnetoelectric [6], Witten [7], or Wormhole effects [13]. We focus instead on a fractional magnetic capacitor, whose implementation and detection are better suited for optical-lattice techniques. Along this route, several intermediate, but interesting, phenomena can be observed in our QS: $i$ ) 3+1 massless Dirac fermions; ii) 3+1 massive Dirac fermions; and iii) Wilson fermions.
We consider a ${ }^{40} \mathrm{~K}$ Fermi gas in an optical superlattice [9], focusing in the Zeeman sublevels (i.e. spins) of the $F=\frac{9}{2}$ hyperfine manifold. Below, we show that laser-assisted tunneling methods lead to the effective Hamiltonian $(\hbar=1)$

$$
H_{\mathrm{eff}}=\sum_{\boldsymbol{r} v} \sum_{\tau \tau^{\prime}} t_{v} c_{\boldsymbol{r}+\boldsymbol{v} \tau^{\prime}}^{\dagger}\left[U_{\boldsymbol{r} \boldsymbol{v}}\right]_{\tau^{\prime} \tau} c_{\boldsymbol{r} \tau}+\text { H.c. }
$$

where $c_{\boldsymbol{r} \tau}^{\dagger}\left(c_{\boldsymbol{r} \tau}\right)$ creates (annihilates) a fermion with spin $\tau$ at site $\boldsymbol{r}=m \hat{\boldsymbol{x}}+n \hat{\boldsymbol{y}}+l \hat{z}$ with $m, n, l \in\{1 \ldots L\}$, and $t_{v} \sim 0.1-1 \mathrm{kHz}$ is the tunneling strength. Here, $U_{\boldsymbol{r} v}$ are operators dressing the hopping from $\boldsymbol{r} \rightarrow \boldsymbol{r}+\boldsymbol{v}, \boldsymbol{v} \in\{\hat{\boldsymbol{x}}, \hat{\boldsymbol{y}}, \hat{\boldsymbol{z}}\}$, and we use gaussian units. These operators usually rely on spin-dependent optical lattices [14]. We use instead spin-independent bichromatic superlattices (Fig. 1a), which trap all levels from the $F=\left\{\frac{7}{2}, \frac{9}{2}\right\}$ manifolds, and allow for lifetimes $\tau_{1} \sim 1 \mathrm{~s}$. The optical potential $V(\boldsymbol{r})=V_{0} \sum_{v}\left[\cos ^{2}\left(\pi r_{v}\right)+\cos ^{2}\left(2 \pi r_{v}\right)\right]$, where $V_{0} \sim 50$ $150 \mathrm{kHz}$ and we have set the lattice spacing to 1 . This yields a cubic superlattice with atoms trapped in the minima at zero energy (i.e. sites), and secondary minima at $\Delta E_{\mathrm{sl}} \sim 50-100$ kHz (i.e. links). The hopping $U_{r v}$ between $F=\frac{9}{2}$ atoms in neighboring sites is mediated by a Raman transition to a $F=\frac{7}{2}$ "bus" level in the intermediate link (Fig. 1a).

Let us consider two states $|\alpha, \zeta\rangle,\left|\alpha^{\prime}, \zeta^{\prime}\right\rangle$, where $\alpha, \alpha^{\prime}$ label the Zeeman sublevels of the $F=\left\{\frac{7}{2}, \frac{9}{2}\right\}$ manifolds, whereas $\zeta, \zeta^{\prime}$ label the band index and center-of-mass coordinates spanning the lattice sites and links. A two-photon process $\left(\omega_{i}\right.$ and $\mathbf{p}_{i}$ are the frequency and momentum of the $i$-th photon), after eliminating an excited level, couples these states

$$
H_{\mathrm{L}}=\sum_{\alpha, \alpha^{\prime} \zeta, \zeta^{\prime}} \sum_{\alpha \zeta} \tilde{\Omega}_{\zeta^{\prime} \zeta^{\prime}}^{\alpha^{\prime}{ }^{\prime} \alpha^{\prime}} c_{\zeta \alpha}+\text { H.c., }, \quad \tilde{\Omega}_{\alpha \zeta}^{\alpha^{\prime} \zeta^{\prime}}=S_{\zeta^{\prime} \zeta} \Omega_{\alpha^{\prime} \alpha} \mathrm{e}^{-\mathrm{i} \omega t}
$$

where $c_{\zeta \alpha}^{\dagger}\left(c_{\zeta \alpha}\right)$ creates (annihilates) a fermion in $|\alpha, \zeta\rangle, S_{\zeta^{\prime} \zeta}$ is the overlap between Wannier wavefunctions in presence of a momentum transfer $\mathbf{p}_{1}-\mathbf{p}_{2}: S_{\zeta^{\prime} \zeta}=\left\langle\zeta^{\prime}\left|e^{-i\left(\mathbf{p}_{2}-\mathbf{p}_{1}\right) \cdot \mathbf{x}}\right| \zeta\right\rangle$, and $\omega=\omega_{1}-\omega_{2}$. The remaining part of the coupling depends on the light polarization and the atomic internal structure. Thus, the formula factors out the contribution of the center-of-mass wavefunction and of the internal degrees of freedom. If the 
transferred momentum is large, we increase the overlap factor $S_{\zeta^{\prime} \zeta}$ between neighboring sites and links, and thus the hopping. Besides, if lasers are far-detuned from this transition, we can adiabatically eliminate the "bus" level, realizing a fourphoton coupling that leads to the single-band Hamiltonian in Eq. (1). Here, the hopping strengths scale as $t_{v} \sim|\Omega|^{2} / d$, where $d \sim 0.2-2 \mathrm{MHz}$, and $\Omega \sim 10-100 \mathrm{kHz}$ is the Rabi frequency (Fig. 1a). Due to the large Zeeman shift $\Delta E_{z} / B \sim 0.3$ $\mathrm{MHz} / \mathrm{G}$, we can independently implement each matrix element of $U_{\boldsymbol{r} v}$ eliminating a different "bus" level. A careful analysis shows that the contributions of other bands and spurious on-site couplings can be neglected [15]. In Figs. 1c-d, we confirm this for two schemes with the necessary ingredients: spin-preserving and spin-flipping hoppings. We use 4 spin components, and design the hopping in terms of Pauli matrices (Fig. 1b), $U_{\boldsymbol{r} \boldsymbol{v}}=\mathrm{e}^{-\mathrm{i} \phi_{v} \alpha_{v}}$, where $\alpha_{v}=\sigma_{z} \otimes \sigma_{v}$ and $\phi_{v} \in \mathbb{R}$. Such block-structure allows the implementation of $U_{r v}$ in parallel for each spin pair, thus reducing the experimental intricacies. The diagonal tunneling can be directly implemented (see Fig 1c). The spin-flipping hopping requires the even/odd sites to be staggered with $\Delta E_{\text {st }} \sim 10-20 \mathrm{kHz}$, but is also efficient (Fig. 1d). This scheme, originally developed for spin-1 bosons with 3-body interactions [15], leads to the interaction picture Hamiltonian in Eq. (1) when the scattering is switched off by Feschbach resonances [16].

Remarkably enough, starting from this ultracold gas of non-relativistic atoms, there are certain regimes where the emergent quasiparticles become ultra-relativistic fermions. We explore such avenues for a translationally-invariant system, where the bulk energy bands come in degenerate pairs $E_{\boldsymbol{k} \pm}=\sum_{v} 2 t_{v} \cos k_{v} \cos \phi_{v} \pm 2\left(\sum_{v} t_{v}^{2} \sin ^{2} k_{v} \sin ^{2} \phi_{v}\right)^{1 / 2}$, and $k \in[-\pi, \pi]^{3}$ lies in the Brillouin zone. In the $\pi$-flux regime $\phi_{v}=\pi / 2$, atoms wandering around plaquettes take on an overall minus sign, and the bands touch at $N_{\mathrm{D}}=8$ different points $\boldsymbol{\Lambda}_{\boldsymbol{d}} \in\left\{\left(d_{x} \pi, d_{y} \pi, d_{z} \pi\right): d_{x}, d_{y}, d_{z}=0,1\right\}$. Around them, low-energy excitations display a relativistic dispersion $E(\boldsymbol{p}) \approx \pm\left(c_{x}^{2} p_{x}^{2}+c_{y}^{2} p_{y}^{2}+c_{z}^{2} p_{z}^{2}\right)^{1 / 2}$, where $c_{v}=2 t_{v}$ is the effective speed of light, and $\mathbf{p}=\mathbf{k}-\boldsymbol{\Lambda}_{\boldsymbol{d}}$. Indeed, imposing an ultraviolet cutoff $\left|p_{v}\right| \leq 1 / 2 \lambda_{c}$, the effective field theory is

$$
H_{\mathrm{eff}}^{d}=\int_{\lambda_{c}} \mathrm{~d}^{3} r \Psi^{\dagger}(\mathbf{r}) H_{\mathrm{D}}^{d} \Psi(\mathbf{r}), H_{\mathrm{D}}^{d}=\sum_{v} c_{v} \alpha_{v}^{d} p_{v},
$$

where $\Psi(\mathbf{r})=\left(c_{1}(\mathbf{r}), c_{2}(\mathbf{r}), c_{3}(\mathbf{r}), c_{4}(\mathbf{r})\right)^{t}$ is the field operator, $\alpha_{v}^{d}=(-1)^{d_{v}} \alpha_{v}$, and $p_{v}=-\mathrm{i} \partial / \partial r_{v}$ is the momentum. The chosen hoppings induce a Clifford algebra $\left\{\alpha_{v}^{d}, \alpha_{\mu}^{d}\right\}=2 \delta_{v \mu}$, and Eq. (3) yields a physical realization of the so-called naive Dirac fermions in LGT [2]. In our scheme, fermion doubling leads to an even number of species which, in contrast to the artificial doublers in LGT, correspond to physical and observable flavors. We remark that each of them has a different chirality $\gamma_{5}^{\boldsymbol{d}}=Q_{5}^{\boldsymbol{d}} \gamma_{5}$, where $\gamma_{5}=\sigma_{z} \otimes \mathbb{I}$, and $Q_{5}^{\boldsymbol{d}}=(-1)^{d_{x}+d_{y}+d_{z}}$ is the axial charge. Chiral symmetry, which plays a fundamental role in the standard model classifying right/left-handed particles $\gamma_{5} \Psi= \pm \Psi$, cannot be incorporated to the lattice globally.

Let us stress that we are not limited to the massless limit, but can also explore a massive regime with $H_{\text {eff }}^{d}+H_{m}$, where

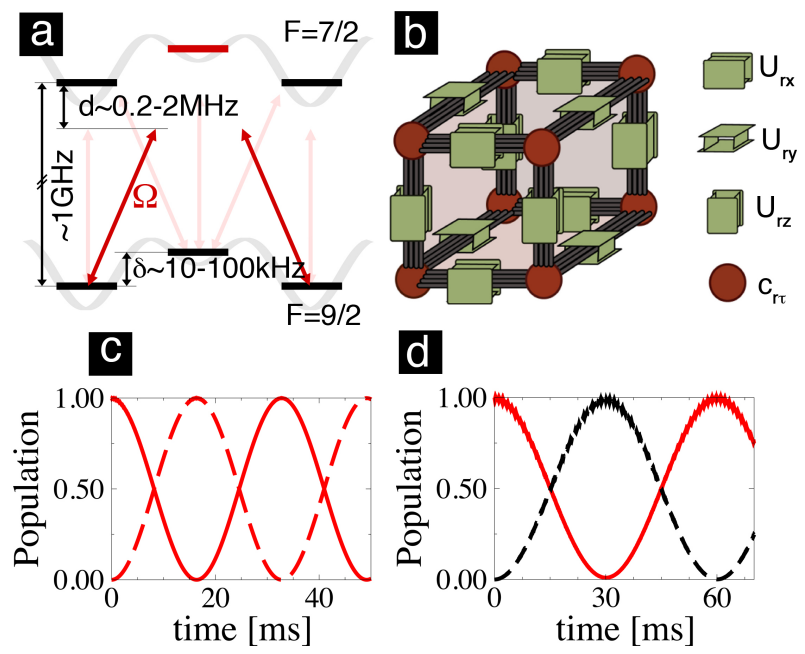

Figure 1: a) Superlattice potential (grey lines) trapping ${ }^{40} \mathrm{~K}$ atoms in the main and secondary minima. The hopping between $F=9 / 2$ levels (in black) is laser-assisted via an intermediate $F=7 / 2$ state (in red). The coupling, detuned by $d+\delta$, is induced by an offresonant Raman transition with Rabi frequency $\Omega$. b) Scheme of the four states of the $F=9 / 2$ manifold (red vertices), connected by laser-induced hoppings (green boxes). c) Time-evolution of the populations of the neighbouring hyperfine levels. The solid (dashed) line is used for site $i(i+1)$; the red (black) line is used for $m_{F}=9 / 2$ $\left(m_{F}=7 / 2\right)$. A clear spin-preserving Rabi oscillation between neighboring sites is shown. The numerical simulation is an exact RungeKutta time-evolution of the complete model involving all the couplings and the levels in Fig. 1a. d) The same as before for a spinipping hopping. Notice the need for a superlattice staggering (10-20 $\mathrm{kHz}$ ) in order to avoid on- site spin-ipping. Exact time-evolution shows oscillations between neighboring sites with a different spin.

$H_{m}=\int \mathrm{d}^{3} r \Psi(\boldsymbol{r})^{\dagger} m c^{2} \beta \Psi(\boldsymbol{r})$, and $\beta=\sigma_{x} \otimes \mathbb{I}$. Since no momentum transfer is required, this term can be engineered via on-site microwave Raman transitions after adiabatic elimination of the $F=\frac{7}{2}$ manifold, where $m c^{2}$ is the Raman strength.

In LGT, Wilson envisaged a method to decouple the doublers from a single Dirac fermion [3]. Our versatile approach allows us to realize his idea by combining the previous ingredients with additional tunnelings $\tilde{U}_{\boldsymbol{r} v}=-\mathrm{i} \mathrm{e}^{-\mathrm{i} \varphi_{v} \beta}$. For $\varphi_{v}=\pi / 2$, the full Hamiltonian in Eq. (3) turns into

$$
H_{\mathrm{eff}}^{\boldsymbol{d}}=\sum_{v} c_{v} \alpha_{v}^{\boldsymbol{d}} p_{v}+m_{\boldsymbol{d}} c^{2} \beta, m_{\boldsymbol{d}}=m-\sum_{v}(-1)^{d_{v}} m_{v}
$$

where $m_{v} c^{2}=2 \tilde{t}_{v}$ depends on the assisted-hopping strength, and thus on the laser power. We highlight that the assisted hopping strengths scale as $\tilde{t}_{v} \sim|\Omega|^{2} / d$. Since $|\Omega|^{2}$ is proportional to the laser intensities, the tunneling strength, and thus the masses, are controlled by the beams power. We finally stress that since $t_{v}, \tilde{t}_{v} \sim 0.1-1 \mathrm{kHz}$, the temperature requirements of this proposal are of the same order as those faced in the context of quantum magnetism in optical lattices, currently at the forefront of experimental research. Setting $m c^{2}=2\left(\tilde{t}_{x}+\tilde{t}_{y}+\tilde{t}_{z}\right)$, doublers become very massive and decouple from the massless fermion at the center of the Brillouin zone $\boldsymbol{\Lambda}_{\mathbf{0}}=\mathbf{0}$. At the expense of breaking chiral symmetry 

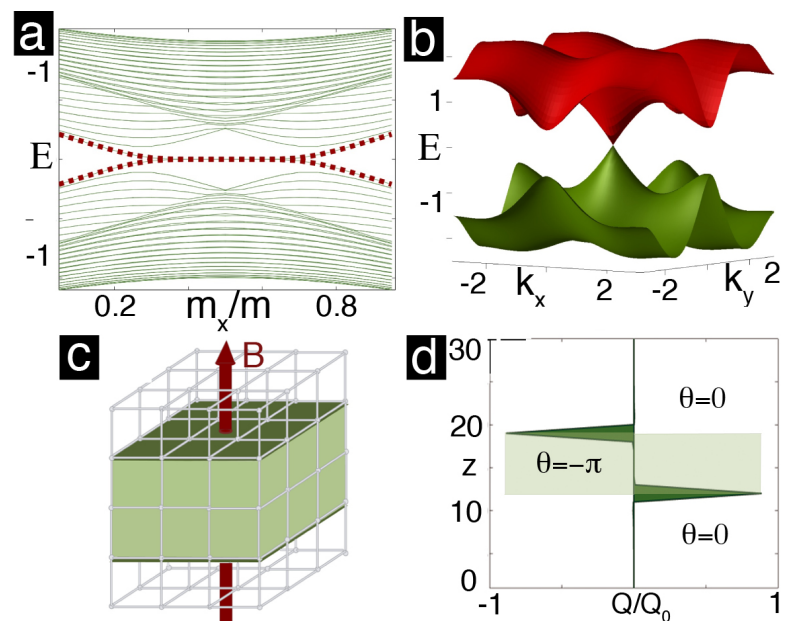

Figure 2: a) In-gap zero-energy modes (dashed red lines) for $\boldsymbol{q}=$ $\left(k_{x}, k_{y}\right)=\mathbf{0}, m / 4 \leq m_{x} \leq 3 m / 4$, and $m_{y}=m / 2, m_{z}=m / 4$, for a lattice with $N=40^{3}$ sites and open boundaries at $z=0, L$. b) Boundary massless Dirac fermion at $z=0, \boldsymbol{q}=\mathbf{0}$, and $m_{x}=m_{y}=m_{z}=m / 2$. c) Scheme for a fractional magnetic capacitor consisting of an axion well: $\boldsymbol{\theta}(\boldsymbol{r}, t)=-\pi$ if $z \in\left[z_{1}, z_{\mathrm{r}}\right]$, and $\boldsymbol{\theta}(\boldsymbol{r}, t)=0$ elsewhere, pierced by a magnetic field $\boldsymbol{B}=B \hat{z}$. This is designed by tuning $m_{x}=m_{y}=m_{z} / 2=m / 4$ globally, whereas $\tilde{m} \gg m$ is only applied to $z_{1}<z<z_{\mathrm{r}}$. d) Accumulated charge on the "plates" of the capacitor, for a lattice of $N=30^{3}$ sites, $m_{x}=m_{y}=m_{z} / 2=m / 4, \tilde{m}=10 m$ (leading to $\theta=-\pi$ for $12<z<18$ ), and flux $\phi / \phi_{0}=2 \pi / 15$.

$\left[H_{\mathrm{D}}^{\boldsymbol{d}}, \gamma_{5}\right] \neq 0$, we have a QS of Wilson fermions invariant under the anti-unitary operator $\mathscr{U}_{a}=\left(\mathrm{iII} \otimes \sigma_{y}\right) \mathscr{K}$, where $\mathscr{K}$ is complex conjugation.

In an effort to preserve chiral symmetry, domain-wall fermions are introduced in 4+1 dimensional LGTs [17], whose lower-dimensional descendants are the so-called topological insulators $[6,7,10,11]$. These holographic phases have an insulating bulk and metallic boundaries where topologicallyprotected midgap Dirac fermions reside. We can realize these phases in experiments by inverting the sign of the Wilson mass through the laser intensity. We study the effect of mass anisotropy on a lattice with open z-boundaries, which leads to the energy spectrum in Fig. 2a. For a critical anisotropy $m_{x}^{c}=\frac{1}{4} m$, a mass inversion occurs and some levels leave the bulk bands to become zero-energy states; whereas for $m_{x}^{c}=\frac{3}{4} m$ the midgap states fuse back into the continuum. In Fig. 2b, the associated energy band displays a solitary $2+1$ massless Dirac fermion exponentially localized around $z=0$, whereas its doubler appears at $z=L$. In other words, there are two distant surfaces with an odd number of massless fermions, an unambiguous signal of a strong TI that defies the doubling.

TIs encode a topological order that has dramatic consequences on their response to electromagnetic fields [6-8]. We explore these effects by synthesizing artificial $\boldsymbol{E}, \boldsymbol{B}$ fields [14], subjected to a modified Gauss law $\nabla \cdot \boldsymbol{E}=4 \pi \rho-\left(e^{2} / \pi c\right) \nabla \theta$. $\boldsymbol{B}$, where $\rho$ is the atomic density. For small masses $[6,7]$, and after a spin rotation, the corresponding Bloch states lead to $\theta=-\frac{\pi}{2} \operatorname{sgn}\left(c_{x} c_{y} c_{z}\right) \sum_{\boldsymbol{d}} Q_{5}^{\boldsymbol{d}} \operatorname{sgn}\left(m_{\boldsymbol{d}}\right)$, which only depends on

the axial charge and the sign of the Wilson mass of each bulk Dirac fermion. In Fig. 3a, one observes that the strong TIs correspond to a non-vanishing axion field $\theta=\pi \bmod (2 \pi)$. Besides, this value is robust with respect to small errors in the implementation of the assisted hopping $c_{v}=c_{v}+\delta c_{v}$.

It is important to notice that the anti-unitary symmetry fixes $\theta=\{0, \pi\}[6]$. However, it is possible to go beyond this scenario [18], and examine further possibilities lying in our proposal, by introducing a complex mass $m \beta+\mathrm{i} \tilde{m} \beta \gamma_{5}$, leading to an arbitrary axion $\theta \in[0,2 \pi)$. Such a term, which breaks $\mathscr{U}_{a}$, is obtained via Raman transitions, and in the isotropic limit leads to

$$
\theta=-\frac{\pi}{2} \sum_{\boldsymbol{d}} Q_{5}^{\boldsymbol{d}} \operatorname{sgn}\left(m_{\boldsymbol{d}}\right)-\sum_{\boldsymbol{d}} Q_{5}^{\boldsymbol{d}} m_{\boldsymbol{d}} \tan ^{-1}\left(\frac{\tilde{m}}{m_{\boldsymbol{d}}}\right),
$$

which contains a perturbation $\delta \theta=-\sum_{d} Q_{5}^{d} m_{\boldsymbol{d}} \tan ^{-1}\left(\tilde{m} / m_{\boldsymbol{d}}\right)$. Note that for $\tilde{m} \rightarrow 0, \delta \theta \rightarrow 0$, we recover the previous result. Conversely, when $\tilde{m} \gg\left|m_{\boldsymbol{d}}\right|$, the transparent expression $\delta \theta=-\frac{\pi}{2} \sum_{d} Q_{5}^{\boldsymbol{d}}\left|m_{\boldsymbol{d}}\right|$ is obtained (Fig. 3b). This perturbation takes on any possible value $\delta \theta \in[-\pi, \pi)$ leading to the total axion of Fig. 3c. Therefore, our atomic gas constitutes a tunable axion medium where the time- and space-dependence of $\theta(\boldsymbol{r}, t)$ can be externally adjusted by tailoring the focusing width and intensity of the Raman lasers $\tilde{m}(\boldsymbol{r}, t)$. We can now explore an exotic consequence of axion electrodynamics: the fractional magnetic capacitor. Engineering the axion medium in Fig. $2 \mathrm{c}$ by focusing a strong Raman laser onto an inner region, confines the axion $\theta=-\pi$ to $z_{1}<z<z_{\mathrm{r}}$, and minimizes the effects of the external trapping potential present in experiments [19]. Besides, we can synthesize a magnetic field generalizing the techniques in [20] to a lattice [14]. The field is introduced via Peierls substitution $t_{v} \rightarrow t_{v} \mathrm{e}^{-\mathrm{i} \int_{v} \mathrm{~d} \boldsymbol{r} \boldsymbol{A}}$ (likewise for $\tilde{t}_{v}$ ) in the Landau gauge $\boldsymbol{A}=-\phi y \hat{\boldsymbol{x}}$, where $\boldsymbol{\phi}$ is the magnetic flux in units of the flux quantum. Setting $\phi=2 \pi p / q$, with $p, q \in \mathbb{Z}$, allows us to diagonalize the open-boundary Hamiltonian in terms of $4 q L$-bands per momentum inside a reduced magnetic Brillouin zone $\boldsymbol{q} \in[-\pi, \pi) \times[-\pi / q, \pi / q)$, and then obtain the density $\rho(z)$ from the occupied eigenstates. Then, the charge per quantum flux is $Q=2 \pi l_{B}^{2} \rho(z)$, where $l_{B}=$ $(c / e B)^{1 / 2}$ is the magnetic length. Subtracting the value in the absence of a magnetic flux leads to Fig. 2d, which confirms the continuum prediction $Q=Q_{0} \delta\left(z-z_{\mathrm{l}}\right)-Q_{0} \delta\left(z-z_{\mathrm{r}}\right)$, where $Q_{0}=\frac{e}{2}$, for an optical lattice with $N=30^{3}$ sites, and magnetic flux $\phi=2 \pi / 15$. In such background, the modified Gauss law predicts an accumulation of fractional charge per flux quantum at the boundaries. Therefore, our axion medium plays the role of an exotic fractional capacitor whose boundaries act as conducting plates. In strong contrast to usual capacitors, the charge stored is fractional, and rather than the electric field, it is the magnetic field which triggers the effect. Let us recall that the ${ }^{40} \mathrm{~K}$ atoms are neutral, and thus this effect corresponds to an accumulation of atomic density rather than charge.

We have described a versatile QS capable of realizing 3+1 massless/massive Dirac fermions, Wilson fermions, and 3D topological insulators as an axion medium. However, we still need to address the detection of such effects. To distinguish 


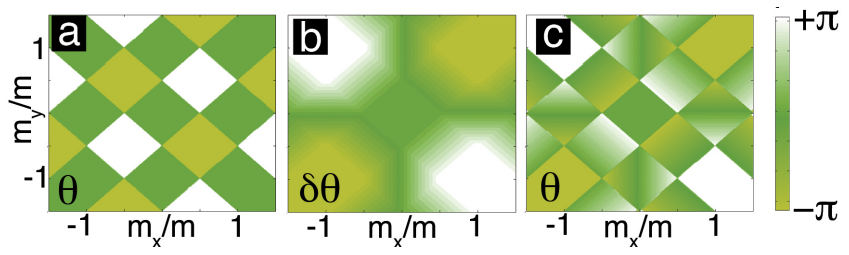

Figure 3: a) Axion index as a function of the masses $m_{y} / m, m_{x} / m$, and setting $m_{z}=m / 2$. In the $\mathscr{U}_{a}$ invariant regime, only fixed values of the axion $\theta=\{0, \pi\}$ are allowed. b) Perturbations to the axion term $\delta \theta$ in the $\mathscr{U}_{a}$-breaking regime. d) Total axion term $\theta$ in the $\mathscr{U}_{a}$-breaking regime.

between the different types of relativistic bulk fermions, it suffices to measure the number of Dirac points at zero energy [21]. This can be accomplished by measuring the atomic density as a function of the chemical potential close to zero energy [22]; a standard technique that relies on absorption images of the Fermi gas (i.e. the shadow projected by the atoms on a CCD camera) after the atoms have been released from the trap. Considerably more challenging is the detection of the edge states characteristic of TIs. The ratio between the number of edge and bulk modes makes the direct detection by density measurements inefficient. Therefore, new but also more demanding methods have been proposed, such as the population of edges modes with bosons [19], or the use of Raman spectroscopy and Bragg scattering [23] . In this Letter, we exploit the consequences of the axion medium to propose a density-based measurement. Let us remark that the accumulation of density in the fractional magnetic capacitor does not suffer from an unbalanced edge-bulk density ratio. In fact, an extensive number of atoms will accumulate on the capacitor plates, which can be detected by phase-contrast imaging methods. These methods do not require the trap release, such as absorption imaging, but rather recover the atomic density in situ by measuring the phase shift of the off-resonant light diffracted by the Fermi gas. Accordingly, phase-contrast imaging is a non-destructive method that has already been implemented for bosons and fermions [24].

In this Letter, we have presented a feasible scheme of laserassisted tunneling in 3D optical lattices, which allows us to design, control, and probe Wilson fermions. This approach appears as a promising route towards the first fully-tunable realization of 3D TIs. We have shown that fractional magnetic capacitors, predicted by axion electrodynamics, can be produced and detected using techniques from optical lattices. Besides, since the axion dynamics is tunable and each boundary can be singled out, phenomena such as the magnetoelectric effect or the boundary fractional quantum Hall effect can also be pursued. Additionally, the interplay between topological order, disorder, and strong interactions can be investigated.

A.B. and M.A.M.D. thank MICINN FIS2009-10061, CAM QUITEMAD, European FET-7 PICC, UCM-BS GICC-
910758 and FPU. M.R. acknowledges EC FP7/2007-2013 (247687, IP AQUTE). N.G. thanks the F.R.S-F.N.R.S. M.L. acknowledges MICINN (FIS2008-00784 and QOIT), EU (NAMEQUAM), ERC (QUAGATUA) and Humboldt Foundation. L.M. and M.R. thank J.I. Cirac and U. Schneider for fruitful discussions in conceiving the superlattice setup.

[1] J.B. Kogut, Rev. Mod. Phys. 55, 775 (1983).

[2] L-H. Karsten and J. Smit, Nuc. Phys. B 183, 103 (1981); H.B. Nielsen and M. Ninomiya, ibid 185, 20 (1981); 193, 173 (1981).

[3] K. Wilson, New Phenomena in Subnuclear Physics. (ed. A. Zichichi, Plenum, New York, 1977).

[4] G. 't Hooft, Phys. Rev. Lett. 37, 8 (1976); R. D. Peccei and H.R. Quinn, ibid. 38, 1440 (1977).

[5] F. Wilczek, Phys. Rev. Lett. 40, 279 (1978); S. Weinberg, ibid 40, 223, (1978); J. Preskill, M.B. Wise, and F. Wilczek, Phys. Lett. B 120, 127 (1983).

[6] X.-L- Qi, T.L. Hughes, and S.-C. Zhang, Phys. Rev. B 78, 195424 (2008); A. M. Essin, J. Moore, and D. Vanderbilt, Phys. Rev. Lett. 102, 146805 (2009).

[7] X.-L Qi et al., Science 323, 1184 (2009); G. Rosenberg, and M. Franz, Phys. Rev. B 82, 035105 (2010).

[8] F. Wilczek, Phys. Rev. Lett. 58, 1799 (1987).

[9] I. Bloch, J. Dalibard, and W. Zwerger, Rev. Mod. Phys. 80, 885 (2008).

[10] M.Z. Hasan, and C.L. Kane, arXiv:1002.3895 .

[11] L. Fu, C.L. Kane, and E.J. Mele, Phys. Rev. Lett. 98, 106803 (2007).

[12] R. Feynman, Int. Jour. of Theor. Phys. 21, 467 (1982).

[13] G. Rosenberg, H.-M. Guo, and M. Franz, Phys. Rev. B 82, 041104 (2010).

[14] D. Jaksch and P. Zoller, New J. Phys. 5, 56 (2003); F. Gerbier and J. Dalibard, ibid 12, 033007 (2010); K. Osterloh et al., Phys. Rev. Lett. 95, 010403 (2005).

[15] L. Mazza, M. Rizzi, M. Lewenstein, and J.I. Cirac, arXiv:1007.2344.

[16] C. Chin, R. Grimm, P. Julienne, and E. Tiesinga, Rev. Mod. Phys. 82, 1225 (2010); G. Roati et al. Nature 453, 895 (2008).

[17] D. B. Kaplan, Phys. Lett. B 288, 342 (1992); M. Creutz and I. Horváth, Phys. Rev. D 50, 2297 (1994).

[18] R. Li, J. Wang, X.-L- Qi, and S.-C. Zhang, Nat. Phys. 6, 284 (2010).

[19] T.D. Stanescu et al., Phys. Rev. A 79, 053639 (2009); T.D. Stanescu, V. Galitski, and S. Das Sarma, Phys. Rev. A 82, 013608 (2010).

[20] Y.-J. Lin et al., Nature 462, 628 (2009).

[21] Massless (massive) naive Dirac fermions have $N_{\mathrm{D}}=8\left(N_{\mathrm{D}}=0\right)$ Dirac points at $E=0$, whereas Wilson fermions have $N_{\mathrm{D}}=1$.

[22] S.-L. Zhu, B. Wang, and L.-M. Duan, Phys. Rev. Lett. 98, 260402 (2007); A. Bermudez et al. New. J. Phys. 12, 033041 (2010).

[23] T.-L. Dao et al., Phys. Rev. Lett. 98, 240402 (2007); X.-J. Liu, X. Liu, C. Wu, and J. Sinova, Phys. Rev. A 81, 033622 (2010).

[24] M.R. Andrews et al., Science 273, 84 (1996); U. Schneider et al., ibid 322, 1520 (2008); Y. Shin, Phys. Rev. Lett. 97, 030401 (2006). 